Kazuhiro Nakayama - Shoji Fukamachi • Hiroshi Kimura

Yoshiro Koda • Augustinus Soemantri • Takafumi Ishida

\title{
Distinctive distribution of AIM1 polymorphism among major human populations with different skin color
}

Received: October 9, 2001 / Accepted: December 3, 2001

\begin{abstract}
The genetic background for human skin color has been a major topic in human genetics; however, its molecular basis is still unclear. The gene for the AIM-1 protein (AIM1) was recently found to be responsible for the body color of medaka fish. In the search for the genes controlling human skin color variations, we have investigated genetic polymorphisms of this gene, and we have found a singlenucleotide polymorphism that has clear association with major human populations in terms of skin color.
\end{abstract}

Key words AIM1 - Melanin synthesis · Population study · Primates $\cdot$ Skin color diversity $\cdot$ SNPs

\section{Introduction}

Among many genes affecting human melanin synthesis, only the melanocortin 1 receptor gene $(M C 1 R)$ can explain nonpathogenic intrapopulation skin color variations; however, interpopulation diversity was not addressed (Flanagan et al. 2000; Harding et al. 2000). Human AIM-1 was first identified as a melanoma antigen with unknown function (Harada et al. 2001). However, recently, Newton et al. (2001) assigned it to the underwhite locus (uw), which

K. Nakayama $\cdot$ T. Ishida $(\triangle)$

Department of Biological Sciences, Graduate School of Science, University of Tokyo, 7-3-1 Hongo, Bunkyo-ku, Tokyo 113-0033, Japan

Tel. +81-3-5841-4633; Fax +81-3-3818-7547

e-mail: tishida@biol.s.u-tokyo.ac.jp

S. Fukamachi

Graduate School of Frontier Science, University of Tokyo, Chiba, Japan

H. Kimura · Y. Koda

Department of Forensic Medicine, Kurume University School of Medicine, Fukuoka, Japan

A. Soemantri

Department of Child Health, Faculty of Medicine, Diponegoro

University, Semarang, Indonesia underlies a new type of oculocutaneous albinism. AIM1 is a newly identified gene in fish that controls melanin synthesis; $B$ (homologue of the human AIM1) mutants of medaka fish displayed hypopigmentation with different phenotypes (Fukamachi et al. 2001). The function of AIM-1 as a transporter in melanin synthesis has been suggested by its 12domain membrane-pass structure, which is similar to the structure of the sucrose transporter in plants (Fukamachi et al. 2001).

We have investigated polymorphisms of human AIMI among representatives of major human populations with varying degrees of skin pigmentation including white South Africans, Ghanaians, Japanese, and New Guinea Islanders to identify any possible relation to skin color variation.

\section{Subjects and methods}

A total of 205 DNA samples from individuals belonging to major human populations, white South Africans $(n=54)$, Ghanaians $(n=50)$, Japanese $(n=49)$, and New Guinea Islanders $(n=52)$, were used in this study. All the anonymously coded samples were collected from unrelated individuals with informed consent. For the comparative study, DNAs from a chimpanzee (Pan troglodytes), a bonobo (Pan paniscus), a gorilla (Gorilla gorilla), an orangutan (Pongo pygmaeus) and a Japanese macaque (Macaca fuscata) were also used.

For the identification of molecular alterations in the AIM1 coding region, primers were designed to amplify seven exons of AIM1 separately (Table 1). After polymerase chain reaction (PCR) amplification, we then determined the nucleotide sequences of each DNA fragment of the PCR product by using an ABI PRISM BigDye Terminator cycle sequencing FS kit and ABI PRISM 310 Genetic Analyzer (Applied Biosystems, Tokyo, Japan).

The genotyping of E272K was performed with a PCRTaqI restriction fragment length polymorphism (RFLP) analysis of exon 3. By using allele-specific forward primers (L374 and F374) and a universal reverse primer (Universal 
R) (Table 1), allele-specific PCR was performed to identify L374F genotypes.

\section{Results and discussion}

In our initial study, we have identified two nonsynonymous single-nucleotide polymorphisms in human AIM1 by determining nucleotide sequences of the coding regions of the gene. They are GAG (glutamate)/AAG (lysine) in codon $272(\mathrm{E} 272 \mathrm{~K})$ and TTG (leucine)/TTC (phenylalanine) in codon 374 (L374F). The distribution of these two substitutions was screened for in the major human populations by using PCR-TaqI RFLP and allele-specific PCR methods. The former substitution, which was found in the database (NCBI dbSNP; rs26722), was polymorphic only in the Japanese and New Guinea Islander populations, and monomorphic (allele frequency: $<0.01$ ) in the white South African and Ghanaian populations. The other single nucleotide substitution causing L374F, which showed a distinctive population distribution, existed exclusively in white South Africans but not in the other populations (Table 2). Since white South Africans are admixtures of several European ethnic groups (Thompson 1990), this substitution is regarded as a Caucasian-type allele. The genetic contribu-

Table 1. Primer sequences

\begin{tabular}{|c|c|c|}
\hline \multirow[t]{2}{*}{ Exon 1} & $\mathrm{~F}$ & 5'-CCAGTTTGAAACACAGACCC-3' \\
\hline & $\mathrm{R}$ & 5'-TCAAACACATGAACATCCTCC-3' \\
\hline \multirow[t]{2}{*}{ Exon 2} & $\mathrm{~F}$ & 5'-GGCAAGAAGTTTAGGTGGAA-3' \\
\hline & $\mathrm{R}$ & 5'-GCTGACCCGTTCATTCA-3' \\
\hline \multirow[t]{2}{*}{ Exon 3} & $\mathrm{~F}$ & 5'-CTGAAGGGGAGTGTCTATGC-3' \\
\hline & $\mathrm{R}$ & 5'-CCCCATGAAACTCTTCTCGT-3' \\
\hline \multirow[t]{2}{*}{ Exon 4} & $\mathrm{~F}$ & 5'-CTTTGTGTGATGGCTGACTG-3' \\
\hline & $\mathrm{R}$ & 5'-GAGGATAGCCCAGAAGAACC-3' \\
\hline \multirow[t]{2}{*}{ Exon 5} & $\mathrm{~F}$ & 5'-GAGGTGGAGAAGCAGAG-3' \\
\hline & $\mathrm{R}$ & 5'-CTGGTATTTTAAACAGTAGGAA-3' \\
\hline \multirow[t]{2}{*}{ Exon 6} & $\mathrm{~F}$ & 5'-TCTTCAGAAGAAACGGATTG-3' \\
\hline & $\mathrm{R}$ & 5'-CCAGCCTTCAGATGAGTC-3' \\
\hline \multirow[t]{2}{*}{ Exon 7} & $\mathrm{~F}$ & 5'-TTTGCTGACCTGTGCCCTAA-3' \\
\hline & $\mathrm{R}$ & 5'-GCAGATTCCACGGCTGAAAT-3' \\
\hline \multicolumn{2}{|l|}{ L374 } & 5'-TTGGATGTTGGGGCTTG-3' \\
\hline \multicolumn{2}{|l|}{ F374 } & 5'-TTGGATGTTGGGGCTTC-3' \\
\hline \multicolumn{2}{|c|}{ Universal R } & 5'-TCССТTTCATTTTCCAGAGA-3' \\
\hline
\end{tabular}

tion of non-Caucasians to the present white South African population (Caucasians) is estimated to be about $7 \%$ from studies of the HLA human major histocompatibility complex (Botha et al. 1975), and this value well accounts for the breakdown of the monomorphic status of Phe (0.89) among the white South Africans in the present study. Newton et al. (2001) also reported that L374F is a common polymorphism.

To identify the prototype of the human allele, we analyzed AIM1 in several primate species (Figure 1), and the TTG (Leucine) allele was deduced to be the prototype. On the basis of partial nucleotide sequences of AIM1 in humans, primates, mouse, and medaka, as well as in the celery sucrose transporter (Lemoine 2000), used as an orthologue, predicted amino acid sequences were aligned and compared (Figure 1). This alignment revealed a highly conserved amino acid sequence throughout all species, from primates to plants, and, especially, the ubiquitous presence of leucine in position 374 of AIM-1. The presence of the leucine residue may be critical for the function of AIM-1.

It is of course premature to presume that the hypopigmentation in the Caucasian population is caused by the loss of leucine in amino acid position 374 of AIM-1. However, not a little genetic evidence suggests an intervention of AIM-1 in mammalian pigmentation. The cytogenetic location of human AIM1 (5p13.3, NCBI LocusLink; 51151) does not preclude AIM1 from being a candidate for an important pigmentation locus, $u w$ (Sweet et al. 1998), and it was, in fact, proved that the AIM1 is responsible for one type of human albinism (Newton et al. 2001). In mice, a double mutation of $u w$ and $M c 1 r$ results in significantly less pigmentation than either mutation alone (Lehman et al. 2000). It is thus speculated that the combination of poly-

Table 2. Allele frequency of L347F

\begin{tabular}{llll}
\hline & & \multicolumn{2}{l}{ Frequency } \\
\cline { 3 - 4 } Population & Number of samples & Leu & Phe \\
\hline White South African & 54 & 0.11 & 0.89 \\
Ghanaian & 50 & 1.00 & 0.00 \\
Japanese & 49 & 1.00 & 0.00 \\
New Guinea Islanders & 52 & 1.00 & 0.00 \\
\hline
\end{tabular}

\begin{tabular}{|c|c|c|c|c|c|c|c|c|c|c|c|c|c|c|c|c|c|c|}
\hline $\mathrm{Ca}$ & $\begin{array}{cc}V & G \\
. & .\end{array}$ & $\begin{array}{l}\text { C } \\
.\end{array}$ & $\begin{array}{l}\text { W } \\
.\end{array}$ & $\begin{array}{l}\mathrm{G} \\
.\end{array}$ & $\begin{array}{l}F \\
L\end{array}$ & $\begin{array}{ll}C & 1 \\
. & . \\
\end{array}$ & I & $\begin{array}{l}\mathrm{N} \\
\cdot \\
\end{array}$ & S & $\begin{array}{l}\mathrm{V} \\
\text {. }\end{array}$ & $\begin{array}{l}\mathrm{F} \\
.\end{array}$ & $\begin{array}{l}S \\
\text {. }\end{array}$ & $\begin{array}{l}S \\
.\end{array}$ & $\begin{array}{l}\mathrm{L} \\
.\end{array}$ & $\begin{array}{l}Y \\
\text {. }\end{array}$ & S & $\begin{array}{l}Y \\
.\end{array}$ & \\
\hline & & & & $\cdot$ & $\mathrm{L}$ & & & & & 7 & & - & $\cdot$ & $\cdot$ & $\cdot$ & & & \\
\hline & & 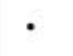 & . & . & & & & & & - & $\cdot$ & - & - & - & - & & & \\
\hline & - & - & . & - & $\mathrm{L}$ & . & . & . & & - & - & - & - & V & - & & - & \\
\hline & - & $\cdot$ & $\cdot$ & $\cdot$ & & 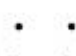 & - & - & $A$ & - & $S$ & $\cdot$ & A & $\cdot$ & $\cdot$ & • & $\cdot$ & \\
\hline elery SUT & - & $S$ & $\mathrm{~L}$ & $\cdot 1$ & L L & $\mathrm{L} \quad \mathrm{L}$ & & $\cdot$ & $\cdot$ & $\cdot$ & $\mathrm{V}$ & $\mathrm{L}$ & G & $\cdot$ & $\mathrm{T}$ & & 1 & \\
\hline
\end{tabular}

Fig. 1. Amino acid alignment of the L347F site and its flanking regions in AIM-1. Great apes used were a chimpanzee, a bonobo, a gorilla, and an orangutan. Mouse and medaka sequences are from the GenBank database, accession numbers AF360357 and AF332510, respectively.
Celery SUT, Apium graveolens sucrose transporter (AgSUT1, GenBank accession number AF063400). Black shading shows the L374F position. In other positions, dots indicate residues identical to the human sequence. Gray shadings show conserved residues 
morphic alleles at two loci, $A I M 1$ and $M C 1 R$, may predict a large part of human skin color diversity.

Acknowledgments This study was partly supported by Grant-in-Aid for Scientific Research on Priority Areas (C) Genome Science from Ministry of Education, Culture, Sports, Science and Technology, Japan.

\section{References}

Botha MC, Campbell E, Briggs B, du Toit ED (1975) Differences on serological definition and frequency of some HLA antigens among southern African populations. In: Kissmeyer-Nielsen F (ed) Histocompatibility testing. Munksgaard, Copenhagen, pp 151-172

Flanagan N, Healy E, Ray A, Philips S, Todd C, Jackson IJ, BrichMachin MA, Rees JL (2000) Pleiotropic effects of the melanocortin 1 receptor $(M C 1 R)$ gene on human pigmentation. Hum Mol Genet 9:2531-2537

Fukamachi S, Shimada A, Shima A (2001) Mutations in the gene encoding B, a novel transporter protein, reduce melanin content in medaka. Nat Genet 28:381-385
Harada M, Li YF, El-Gamil M, Rosenberg SA, Robbins PF (2001) Use of an in vitro immunoselected tumor line to identify shared melanoma antigens recognized by HLA-A *0201-restricted T cells. Cancer Res 61:1089-1094

Harding RM, Healy E, Ray AJ, Ellis NS, Flanagan N, Todd C, Dixon C, Sajantila A, Jackson IJ, Birch-Machin MA, Rees JL (2000) Evidence for variable selective pressure at MC1R. Am J Hum Genet 66:1351-1361

Lehman AL, Silvers WK, Puri N, Wakamatsu K, Ito S, Brilliant MH (2000) The underwhite (uw) locus acts autonomously and reduces the production of melanin. J Invest Dermatol 115:601-606

Lemoine R (2000) Sucrose transporters in plants: update on function and structure. Biochim Biophys Acta 1465:246-262

Newton JM, Cohen-Barak O, Hagiwara N, Gardner JM, Davission MT, King RA, Brilliant MH (2001) Mutations in the human orthologue of the mouse underwhite gene (uw) underlie a new form of oculocutaneous albinism, OCA4. Am J Hum Genet 69:981988

Sweet HO, Brilliant MH, Cook SA, Johnson KR, Davisson MT (1998) A new allelic series for the underwhite gene on mouse chromosome 15. J Hered 89:546-551

Thompson L (1990) A history of South Africa. Yale University Press, New Haven, CT, USA, pp 31-109 